\title{
Risk scores as useful predictors of perioperative complications in patients with rectal cancer who received radical surgery
}

\author{
Hiroshi Miyakita ${ }^{1} \cdot$ Sotaro Sadahiro $^{1} \cdot$ Gota Saito $^{1} \cdot$ Kazutake Okada $^{1} \cdot$ \\ Akira Tanaka $^{1}$ - Toshiyuki Suzuki ${ }^{1}$
}

Received: 29 June 2016 / Accepted: 9 October 2016 / Published online: 25 October 2016

(C) The Author(s) 2016. This article is published with open access at Springerlink.com

\begin{abstract}
Background Rectal cancer is associated with a higher rate of surgical complications. The ability to predict the risk of complications before treatment would facilitate the design of personalized treatment strategies optimally suited for each patient.

Methods We retrospectively studied 260 patients with rectal cancer who underwent radical surgery to examine the relations between complications and 5 types of risk scores. Results Complications developed in 56 patients $(21.5 \%)$. Nineteen patients had infectious complications, 16 had intestinal obstruction, and 12 had other complications. Twelve patients out of 187 patients who received low anterior resection had anastomotic leakage. Estimation of Physiologic Ability and Surgical Stress Comprehensive Risk Score (E-PASS CRS) and Neutrophil-to-lymphocyte Ratio (NLR) were significantly related to all complications, infectious complications, and anastomotic leakage. Surgical Apgar Score was significantly related to infectious complications. Prognostic Nutritional Index was significantly related to all complications and intestinal obstruction. Colorectal Physiologic and Operative Severity Score for the Enumeration of Mortality and Morbidity was significantly related to all complications, and infectious complications. A multivariate analysis showed that body-mass index, E-PASS CRS, and NLR were independent risk factors for anastomotic leakage. In particular, NLR was the only score that could be evaluated before surgery.
\end{abstract}

Sotaro Sadahiro

sadahiro@is.icc.tokai.ac.jp

1 Department of Surgery, School of Medicine, Tokai

University, 143 Shimokasuya, Isehara, Kanagawa 259-1193,

Japan
Conclusions Five types of risk scores were useful methods for evaluating the risks of complications in patients with rectal cancer. NLR is a score that can be evaluated before surgery and predicted the risk of anastomotic leakage, suggesting that it is useful for assessing the need for a diverting colostomy.

Keywords Rectal cancer - Complication · Anastomotic leakage $\cdot$ Risk score

\section{Introduction}

Colorectal cancer is the third most common malignant disease and ranks as the third leading cause of cancer-related death [1]. The standard treatment for colorectal cancer is surgical resection. Patients with postoperative complications have been reported to have poor long-term outcomes [2, 3]. The perioperative complications is associated with not only short-term disadvantages such as a compromised quality of life, but also with increased medical costs, delayed initiation of postoperative adjuvant chemotherapy [4], high recurrence rates [5], and poor long-term outcomes [5-7]. Rectal cancer has higher incidences of infectious complications and anastomotic leakage than colon cancer [8, 9]. In particular, lower rectal cancer is an important risk factor for anastomotic leakage. The development of anastomotic leakage has been reported to increase the rate of local recurrence [10]. The international standard treatment for rectal cancer is multidisciplinary treatment, including preoperative chemoradiotherapy [11]. Surgical procedures can be selected from a number of options, including sphincter-preserving surgery, transanal local excision, and defunctioning stomas. The ability to predict the risk of complications before treatment would most likely facilitate selection of the treatment policy optimally suited for individual patients. 
To date, various risk scores have been proposed for patients to undergo elective surgery, including the Estimation of Physiologic Ability and Surgical Stress Comprehensive Risk Score (E-PASS CRS) [12], Surgical Apgar Score (SAS) [13], the Prognostic Nutritional Index (PNI) proposed by Onodera et al. [14], the Neutrophil-to-lymphocyte Ratio (NLR) [15], and the Physiological and Operative Severity Score for the Enumeration of Mortality and Morbidity (POSSUM) [16]. In addition, Colorectal-POSSUM (CR-POSSUM) [19] has been proposed for patients to undergo elective surgery for colorectal cancer. In the present study, we evaluated risk scores as a means of predicting perioperative complications in patients who underwent radical surgery for rectal cancer.

\section{Patients and methods}

From January 2003 through December 2013, a total of 392 patients underwent radical surgery for rectal adenocarcinoma in our hospital. We excluded 131 patients who underwent emergency surgery or laparoscopic surgery and studied the remaining 260 patients. Data on these patients were retrospectively collected to estimate the incidences of complications within 30 days after surgery and to compare the value of each risk score for predicting the probability of complications.

\section{Risk evaluation scores}

We studied the following risk evaluation scores: Estimation of Physiologic Ability and Surgical Stress Comprehensive Risk Score (E-PASS CRS) [12], Surgical Apgar Score (SAS) [13], Prognostic Nutritional Index (PNI) [14], Colorectal POSSUM (CR-POSSUM) [16], and Neutrophilto-lymphocyte Ratio (NLR) [15]. The preoperative general condition, concomitant diseases, and complications of each patient were examined from their medical records. Surgical information, such as intraoperative vital signs and bleeding volume, was obtained from each patient's surgical and anesthesiologic records.

E-PASS CRS was calculated as described by Haga et al. [12]. The preoperative risk score, reflecting the patient's physiological status before surgery, the surgical stress score, reflecting the degree of surgical invasion, and the comprehensive risk score, representing the overall risk associated with preoperative risk and surgical stress, were calculated for each patient. SAS was calculated from the intraoperative bleeding volume, the minimal heart rate, and the minimal mean blood pressure, as described by Gawande et al. [13]. PNI was calculated by the following formula, proposed by Onodera et al. [14]: PNI $=10 \times$ serum albumin level $(\mathrm{g} /$ $\mathrm{dL})+0.05 \times$ total lymphocyte count $\left(\mathrm{mm}^{3}\right)$. CR-POSSUM was calculated as reported by Tekkis et al. $[16,17]$ on the basis of the Physiological Score (PS), derived from age and the results of preoperative assessments of cardiac dysfunction, systolic blood pressure, heart rate, serum hemoglobin concentration, and urea nitrogen concentration, and the Operative Severity Score (OS), derived from surgical invasion, Duke's classification, and intraoperative findings. The CR-POSSUM score was the sum of PS and OS. NLR was calculated using blood samples obtained at initial presentation. In patients who received preoperative chemoradiotherapy, the score was calculated before chemoradiotherapy.

\section{Classification and severity of complications}

We studied the following 4 types of complications occurring within 30 days after surgery: all complications, infectious complications (wound infection, inflammation of the pelvic dead space, and intraabdominal abscess), anastomotic leakage, and intestinal obstruction. All complications included exacerbation of underlying disease. The diagnosis of anastomotic leakage was based on the properties of drainage fluid or radiographic findings. The severity of complications was evaluated according to the ClavienDindo classification [18]. All complications, infectious complications, and intestinal obstruction of Clavien-Dindo grade $3 \mathrm{a}$ or higher that required surgical intervention and anastomotic leakage of Clavien-Dindo grade $3 b$ or higher that required reoperation were defined as complications.

\section{Evaluation of risk factors for anastomotic leakage}

To investigate the risk factors for anastomotic leakage, we excluded 76 patients with a diverting stoma at initial surgery from the 187 patients who underwent low anterior resection and studied the remaining 111 patients. To compare the accuracy of each score for predicting the risk of anastomotic leakage, we calculated the sensitivity, specificity, positive predictive value (PPV), negative predictive value (NPV), and accuracy rate for each score. We then compared the values among the different scores. A multivariate analysis was then performed to identify risk factors for anastomotic leakage in patients without a diverting stoma at initial surgery. The model included factors that were significantly related to anastomotic leakage in our study, as well as sex, bodymass index (BMI), smoking history, the American Society of Anesthesiologists (ASA) classification, tumor location, and preoperative chemoradiotherapy, ypStage, which have been reported to be risk factors for anastomotic leakage in patients with rectal cancer $[19,20]$.

\section{Statistical analysis}

The cutoff value (COV) for each score was calculated by risk evaluation analysis, performed using receiver operating 
Table 1 Patients' characteristics

\begin{tabular}{|c|c|}
\hline Variable & $n(\%)$ \\
\hline \multicolumn{2}{|l|}{ Sex } \\
\hline Male & $190(73)$ \\
\hline Female & $70(28)$ \\
\hline \multicolumn{2}{|l|}{ Age (year) } \\
\hline Range & $28-92$ \\
\hline Median & 63 \\
\hline \multicolumn{2}{|l|}{ Location of the tumor } \\
\hline Upper and middle & $112(43)$ \\
\hline Lower & $148(57)$ \\
\hline \multicolumn{2}{|l|}{ Neoadjuvant CRT } \\
\hline Yes & $202(77)$ \\
\hline No & $58(23)$ \\
\hline \multicolumn{2}{|c|}{ Concurrent chemotherapy } \\
\hline UFT & $37(19)$ \\
\hline S-1 & $165(81)$ \\
\hline \multicolumn{2}{|l|}{ Radiation } \\
\hline 40 or $45 \mathrm{~Gy}$ & $183(91)$ \\
\hline 20 Gy and IOR & $19(9)$ \\
\hline \multicolumn{2}{|l|}{ Surgical procedure } \\
\hline LAR & $187(70)$ \\
\hline APR & $73(30)$ \\
\hline \multicolumn{2}{|l|}{ Histological type } \\
\hline Well & $129(50)$ \\
\hline Moderate & $92(35)$ \\
\hline Poor & $1(0.3)$ \\
\hline Mucinous & $12(5)$ \\
\hline $\mathrm{pCR}$ & $26(10)$ \\
\hline \multicolumn{2}{|l|}{ Lymphatic invasion } \\
\hline Positive & $121(46)$ \\
\hline Negative & $138(53)$ \\
\hline Unknown & $1(0.3)$ \\
\hline \multicolumn{2}{|l|}{ Venous invasion } \\
\hline Positive & $126(48)$ \\
\hline Negative & $133(51)$ \\
\hline Unknown & $1(0.3)$ \\
\hline \multicolumn{2}{|c|}{ Pathological stage (include ypStage) } \\
\hline 0 & $29(11)$ \\
\hline I & $79(30)$ \\
\hline II & $78(31)$ \\
\hline III & $73(28)$ \\
\hline
\end{tabular}

$C R T$ chemoradiotherapy; LAR: low anterior resection; APR abdominoperineal resection; $p C R$ pathological complete response

characteristic curves (ROC) in which the presence of complications was considered a positive result. The patients were divided into 2 groups according to whether their score was less than the COV or equal to or greater than the COV, and the incidence of complications was compared. For risk
Table 2 Postoperative complications according to the Cravien-Dindo grade

\begin{tabular}{llc}
\hline Complication & C-D grade & $n(\%)$ \\
\hline Infectious complication & $3 \mathrm{a}$ & $18(27.6)$ \\
& $3 \mathrm{~b}$ & $1(1.5)$ \\
Anastomotic leakage & 4 & $0(0)$ \\
& $3 \mathrm{a}$ & $6(27.2)$ \\
Bowel obstruction & $3 \mathrm{~b}$ & $11(55.0)$ \\
& 4 & $1(5.0)$ \\
& $3 \mathrm{a}$ & $8(26.6)$ \\
& $3 \mathrm{~b}$ & $8(26.6)$ \\
\hline
\end{tabular}

$C-D$ grade Cravien-Dindo grade

evaluation analysis, the COV of the PNI was set at 40 , as recommended by Onodera et al. [14]. The 2 groups were compared with the use of the Chi square test. Multiple logistic regression analysis was performed. $P$ values of less than 0.05 were considered to indicate statistical significance. All statistical analyses were performed with the use of JMP ${ }^{\circledR} 10$ software (SAS Institute Inc., Cary, NC, USA).

This study was approved by the institutional review board of Tokai University (15R-217).

\section{Results}

The patients' characteristics are shown in Table 1. The surgical procedure was lower anterior resection (LAR) in 187 patients and abdominoperineal resection (APR) in 73 patients. A total of 202 patients $(77 \%)$ received preoperative chemoradiotherapy.

We used preoperative chemoradiotherapy for patients with clinical Stage II or III locally advanced rectal adenocarcinoma according to the NCCN guideline [11]. Tumor location was defined according to the Japanese criteria. The detail was reported previously [21].

One or more complication developed in 56 patients $(21.5 \%)$. Nineteen patients $(7.3 \%)$ had infectious complications, $16(6.1 \%)$ had intestinal obstruction, and 12 (4.6\%) had other complications. Anastomotic leakage was occurred in 12 patients $(10.8 \%)$ out of 111 patients who received low anterior resection without diverting stomas (Table 2).

\section{Evaluation of risk scores and the incidences of complications (Table 3)}

E-PASS CRS was significantly related to the incidences of all complications, infectious complications, and anastomotic leakage. PNI was significantly related to the 
Table 3 Relation between the predictive scoring systems and the incidence of complication

\begin{tabular}{|c|c|c|c|c|c|}
\hline Complication & Cut-off value & Incidence $(\%)$ & OR & $95 \% \mathrm{CI}$ & $p$ value \\
\hline \multicolumn{6}{|l|}{ E-PASS CRS } \\
\hline \multirow[t]{2}{*}{ All complication } & $<0.294$ & 16/134 (11.9) & Reference & & \\
\hline & $\geq 0.294$ & $40 / 126(31.7)$ & 3.45 & $1.84-6.73$ & $<0.0001$ \\
\hline \multirow[t]{2}{*}{ Infectious complication } & $<0.294$ & $5 / 134(3.7)$ & Reference & & \\
\hline & $\geq 0.294$ & 14/126 (11.1) & 3.23 & $1.19-10.23$ & 0.0202 \\
\hline \multirow[t]{2}{*}{ Anastomotic leakage } & $<0.294$ & 4/72 (5.5) & Reference & & \\
\hline & $\geq 0.294$ & $8 / 39(20.5)$ & 4.38 & $1.28-17.46$ & 0.0183 \\
\hline \multirow[t]{2}{*}{ Bowel obstruction } & $<0.294$ & $5 / 134(3.7)$ & Reference & & \\
\hline & $\geq 0.294$ & $11 / 126(8.7)$ & 2.46 & $0.86-8.02$ & 0.0906 \\
\hline \multicolumn{6}{|l|}{ PNI } \\
\hline \multirow[t]{2}{*}{ All complication } & $\geq 41$ & 29/179 (16.1) & Reference & & \\
\hline & $<40$ & 27/81 (33.3) & 2.60 & $1.41-4.80$ & 0.0022 \\
\hline \multirow[t]{2}{*}{ Infectious complication } & $\geq 41$ & $12 / 179(6.7)$ & Reference & & \\
\hline & $<40$ & 7/81 (8.6) & 1.32 & $0.47-3.42$ & 0.5750 \\
\hline \multirow[t]{2}{*}{ Anastomotic leakage } & $\geq 41$ & $8 / 85(9.4)$ & Reference & & \\
\hline & $<40$ & $4 / 26(15.4)$ & 1.75 & $0.48-6.35$ & 0.3907 \\
\hline \multirow[t]{2}{*}{ Bowel obstruction } & $\geq 41$ & 7/179 (3.9) & Reference & & \\
\hline & $\geq 40$ & 9/81 (11.1) & 3.08 & $1.10-8.94$ & 0.0311 \\
\hline \multicolumn{6}{|l|}{ NLR } \\
\hline \multirow[t]{2}{*}{ All complication } & $<2.21$ & $17 / 123(13.8)$ & Reference & & \\
\hline & $\geq 2.21$ & $39 / 137(28.5)$ & 2.50 & $1.35-4.81$ & 0.0033 \\
\hline \multirow[t]{2}{*}{ Infectious complication } & $<2.21$ & $4 / 123(3.2)$ & Reference & & \\
\hline & $\geq 2.21$ & 15/137 (10.9) & 3.65 & $1.28-13.11$ & 0.0138 \\
\hline \multirow[t]{2}{*}{ Anastomotic leakage } & $<2.21$ & $10 / 62(16.1)$ & Reference & & \\
\hline & $\geq 2.21$ & $2 / 49(4.08)$ & 4.51 & $1.11-30.38$ & 0.0329 \\
\hline \multirow[t]{2}{*}{ Bowel obstruction } & $<2.21$ & $5 / 123(4.0)$ & Reference & & \\
\hline & $\geq 2.21$ & $11 / 137(8.0)$ & 2.06 & $0.69-6.10$ & 0.1842 \\
\hline \multicolumn{6}{|l|}{ Surgcal apgar score } \\
\hline \multirow[t]{2}{*}{ All complication } & $\geq 5$ & $48 / 239(20.0)$ & Reference & & \\
\hline & $<5$ & $8 / 21(38.1)$ & 2.46 & $0.92-6.18$ & 0.0692 \\
\hline \multirow[t]{2}{*}{ Infectious complication } & $\geq 5$ & $14 / 239(5.8)$ & Reference & & \\
\hline & $<5$ & $5 / 21(23.8)$ & 5.02 & $1.47-15.09$ & 0.0119 \\
\hline \multirow[t]{2}{*}{ Anastomotic leakage } & $\geq 5$ & 12/108 (11.1) & & & \\
\hline & $<5$ & $0 / 3$ & - & - & 0.5410 \\
\hline \multirow[t]{2}{*}{ Bowel obstruction } & $\geq 5$ & $15 / 239(6.3)$ & Reference & & \\
\hline & $<5$ & $1 / 21(4.8)$ & 0.74 & $0.09-5.94$ & 0.7819 \\
\hline \multicolumn{6}{|l|}{ CR-POSSUM } \\
\hline \multirow[t]{2}{*}{ All complication } & $<18$ & $18 / 124(14.5)$ & Reference & & \\
\hline & $\geq 18$ & 38/136 (27.9) & 2.26 & $1.22-4.29$ & 0.0086 \\
\hline \multirow[t]{2}{*}{ Infectious complication } & $<18$ & $3 / 124(2.4)$ & Reference & & \\
\hline & $\geq 18$ & 16/136 (11.8) & 5.37 & $1.52-18.93$ & 0.0038 \\
\hline Anastomotic leakage & $<18$ & $7 / 75(9.3)$ & Reference & & \\
\hline & $\geq 18$ & $5 / 36(13.8)$ & 1.30 & $0.37-4.24$ & 0.6660 \\
\hline Bowel obstruction & $<18$ & $5 / 124(4.0)$ & Reference & & \\
\hline & $\geq 18$ & $11 / 136(8.1)$ & 2.09 & $1.73-6.81$ & 0.1681 \\
\hline
\end{tabular}

OR odds ratio; 95\% CI 95\% confidence interval; E-PASS CRS Estimation of Physiologic Ability and Surgical Stress Comprehensive Risk Score; SAS Surgical Apgar Score; PNI Onodera's prognostic nutritional index; NLR neutrophilic lymphocytes ratio; CR-POSSUM colorectal physiological and operative severity score for the enumeration of mortality and morbidity 
incidences of all complications and intestinal obstruction. NLR was significantly related to the incidences of all complications, infectious complications, and anastomotic leakage. SAS was significantly related to the incidence of infectious complications. CR-POSSUM was significantly related to the incidences of all complications, infectious complications, and intestinal obstruction.

\section{Evaluation of risk factors for anastomotic leakage}

Univariate analysis showed that E-PASS CRS and NLR were risk factors related to anastomotic leakage (Table 4). The ASA classification is included in E-PASS CRS and was therefore excluded. A multivariate analysis was performed, including 8 variables, i.e., 6 variables that have

Table 4 Univariate analysis of anastomotic leakage

\begin{tabular}{|c|c|c|c|}
\hline Variable & $\begin{array}{l}\text { Patients } \\
\text { with leakage }\end{array}$ & $\begin{array}{l}\text { Patients without } \\
\text { leakage }\end{array}$ & $p$ value \\
\hline \multicolumn{4}{|l|}{ Sex } \\
\hline Male & 11 & 122 & \\
\hline Female & 1 & 53 & 0.1045 \\
\hline \multicolumn{4}{|l|}{ Age } \\
\hline Range & $43-77$ & $28-92$ & \\
\hline Median & 64.5 & 63 & 0.5691 \\
\hline \multicolumn{4}{|l|}{ BMI } \\
\hline$\geq 25$ & 4 & 32 & \\
\hline$<25$ & 8 & 143 & 0.2009 \\
\hline \multicolumn{4}{|l|}{ Smoking history } \\
\hline No & 4 & 86 & \\
\hline Yes & 8 & 89 & 0.3168 \\
\hline \multicolumn{4}{|c|}{ Location of the tumor } \\
\hline Upper or middle & 7 & 104 & \\
\hline Lower & 5 & 71 & 0.9404 \\
\hline \multicolumn{4}{|l|}{ pStage } \\
\hline 0 & 4 & 21 & \\
\hline I & 3 & 54 & \\
\hline II & 2 & 49 & \\
\hline III & 3 & 51 & 0.2083 \\
\hline \multicolumn{4}{|l|}{ CRT } \\
\hline No & 2 & 40 & \\
\hline Yes & 10 & 135 & 0.6191 \\
\hline \multicolumn{4}{|l|}{ E-PASS CRS } \\
\hline$<0.294$ & 4 & 104 & \\
\hline$\geq 0.294$ & 8 & 71 & 0.0767 \\
\hline \multicolumn{4}{|l|}{ NLR } \\
\hline$<2.21$ & 2 & 86 & \\
\hline$\geq 2.21$ & 10 & 89 & 0.0292 \\
\hline
\end{tabular}

$B M I$ body mass index, CRT chemoradiotherapy, E-PASS CRS Estimation of Physiologic Ability and Surgical Stress Comprehensive Risk Score, $N L R$ neutrophilic lymphocytes ratio been reported to be risk factors for suture failure in patients with rectal cancer (sex, BMI, smoking status, tumor location, pStage, and the presence or absence of preoperative chemoradiotherapy) in addition to E-PASS CRS and NLR. The results showed that E-PASS CRS ( $p=0.0075$, odds ratio $=6.85)$, and NLR $(p=0.0089$, odds ratio $=8.24)$ were independent risk factors for anastomotic leakage (Table 5).

The sensitivity, specificity, PPV, NPV, and accuracy rate of the 5 scores for the prediction of anastomotic leakage were calculated (Table 6). E-PASS CRS and NLR had higher PPV, NPV, and accuracy rates than the other scores.

\section{Discussion}

The development of perioperative complications in patients with rectal cancer has been reported to delay the start of adjuvant chemotherapy [4], potentially leading to poor

Table 5 Multivariate logistic regression analysis of anastomotic leakage

\begin{tabular}{|c|c|c|c|}
\hline & OR & $95 \% \mathrm{CI}$ & $p$ value \\
\hline \multicolumn{4}{|l|}{ Sex } \\
\hline Female & Reference & & \\
\hline Male & 3.66 & $0.60-71.08$ & 0.1778 \\
\hline BMI & 1.31 & $1.02-1.72$ & 0.0775 \\
\hline \multicolumn{4}{|l|}{ Smoking history } \\
\hline No & Reference & & \\
\hline Yes & 1.76 & $0.47-7.72$ & 0.4018 \\
\hline \multicolumn{4}{|c|}{ Location of the tumor } \\
\hline Upper or middle & Reference & & \\
\hline Lower & 1.59 & $0.42-6.61$ & 0.4903 \\
\hline \multicolumn{4}{|l|}{ CRT } \\
\hline No & Reference & & \\
\hline Yes & 2.13 & $0.42-16.82$ & 0.3808 \\
\hline \multicolumn{4}{|l|}{ pStage } \\
\hline 0 & Reference & & \\
\hline 1 & 5.44 & $0.59-60.06$ & 0.1317 \\
\hline 2 & 7.60 & $0.90-84.88$ & 0.0617 \\
\hline 3 & 4.92 & $0.61-48.42$ & 0.1322 \\
\hline \multicolumn{4}{|l|}{ E-PASS CRS } \\
\hline$<0.294$ & Reference & & \\
\hline$\geq 0.294$ & 6.85 & $1.63-39.63$ & 0.0075 \\
\hline \multicolumn{4}{|l|}{ NLR } \\
\hline$<2.21$ & Reference & & \\
\hline$\geq 2.21$ & 8.24 & $1.61-76.07$ & 0.0089 \\
\hline
\end{tabular}

OR odds ratio, $95 \%$ CI $95 \%$ confidence interval, BMI body mass index, CRT chemoradiotherapy, E-PASS CRS Estimation of Physiologic Ability and Surgical Stress Comprehensive Risk Score, NLR neutrophilic lymphocytes ratio 
Table 6 Accuracy rate of anastomotic leakage according to predictive scoring systems

\begin{tabular}{llllllllrr}
\hline Score & COV & OR & $95 \%$ CI & $p$ value & Accuracy rate $(\%)$ & Sensitivity $(\%)$ & Specificity $(\%)$ & PPV (\%) & NPV $(\%)$ \\
\hline E-PASS CRS & 0.294 & 4.38 & $1.28-17.46$ & 0.0183 & 68.4 & 66.6 & 68.6 & 20.5 & 94.4 \\
PNI & 40 & 1.75 & $0.48-6.35$ & 0.3907 & 27.0 & 66.6 & 22.2 & 9.4 & 84.6 \\
NLR & 2.21 & 4.51 & $1.11-30.38$ & 0.0329 & 51.3 & 83.3 & 47.4 & 16.1 & 95.9 \\
SAS & 5 & 0 & - & 0.5410 & 13.5 & 100 & 96.9 & 11.0 & 100 \\
CR-POSSUM & 18 & 1.30 & $0.37-4.24$ & 0.6660 & 34.2 & 58.3 & 31.3 & 9.3 & 86.1 \\
\hline
\end{tabular}

$C O V$ cut off value; $O R$ odds ratio; $95 \%$ CI 95\% confidence interval; $P P V$ positive predictive value; $N P V$ negative predictive value; $E$ - $P A S S C R S$ Estimation of Physiologic Ability and Surgical Stress Comprehensive Risk Score; SAS Surgical Apgar Score; PNI Onodera's prognostic nutritional index; NLR neutrophilic lymphocytes ratio; CR-POSSUM Colorectal physiological and operative severity score for the enumeration of mortality and morbidity

long-term outcomes [5-7]. The ability to predict the likelihood of postoperative complications before starting treatment would thus facilitate the design of personalized treatment strategies for individual patients, including the selection of surgical procedures such as diverting colostomy.

Complications following rectal cancer surgery consisted several categories, such as cardiovascular, respiratory, urinary, wound infection, intraabdominal abscess and anastomotic leakage. However, we selected infectious complications, anastomotic leakage, intestinal obstruction and overall complications in the present study.

E-PASS is a severity score quantifying general condition and surgical risk. It has been reported to be related to postoperative complications and overall survival in elderly patients with colon cancer and those with gastric cancer [22, 23]. Haga et al. reported that E-PASS is useful for predicting the risk of anastomotic leakage in patients who have undergone gastrointestinal surgery [24, 25]. The blood lymphocyte count is an index of immune status that is used to calculate several scores. The PNI proposed by Onodera et al. is calculated from the serum albumin concentration and lymphocyte count and is a useful index of immune and nutritional status in patients with gastrointestinal cancer [14]. Patients with colorectal cancer and a low PNI have a poor prognosis [26]. A PNI of less than the COV of 45.5 has been reported to be an independent risk factor for serious complications, such as myocardial infarction and pulmonary embolism [27].

NLR is a useful prognostic factor in patients with colorectal cancer [15, 28]. A low NLR before surgery is related to disease-free survival and overall survival [29, 30]. NLR on postoperative day 1 is a risk factor for infectious complications [31, 32]. SAS is related to surgical outcomes and is calculated on the basis of bleeding volume, intraoperative minimal blood pressure, and minimal heart rate, and is thus simpler to use than CR-POSSUM and E-PASS. Patients with a high SAS after colectomy have a low incidence of complications after discharge within 30 days after surgery
[33]. The modified Surgical Apgar Score (mSAS), which uses a different COV for intraoperative bleeding volume from the SAS, has been reported to be useful for predicting complications after gastrectomy [34].

CR-POSSUM is a modified score based on POSSUM [11], a severity score that quantifies general condition and surgical risk in patients with colorectal disease. CRPOSSUM is useful for predicting the risk of death within 30 days after surgery for colorectal cancer [17]. In our study, E-PASS CRS, SAS, PNI, NLR, and CR-POSSUM were useful methods for evaluating the risk of complications in patients who underwent radical surgery for rectal cancer. A multivariate analysis was performed including the 8 variables of E-PASS CRS and NLR, found to be significantly related to anastomotic leakage in our study, as well as sex, BMI, smoking history, tumor location, and the presence or absence of preoperative chemoradiotherapy, pStage which that have been reported to be risk factors for anastomotic leakage in patients with rectal cancer. The results showed that E-PASS CRS, and NLR were independent risk factors for anastomotic leakage. McDermott et al. reported co-morbidity is a risk factor for colorectal anastomotic leakage [19]. E-PASS CRS is calculated from factors including co-morbidity. NLR has not been reported to associate with anastomotic leakage in colorectal cancer up to now.

In addition, E-PASS CRS and NLR had higher PPV, NPV, and accuracy rates than the other scores.

All 5 evaluation scores assessed in our study can be calculated from general laboratory data and surgical course. However, E-PASS CRS, SAS, and CR-POSSUM include variables measured during surgery and therefore cannot be calculated until after surgery. Only PNI and NLR can be used to preoperatively evaluate risk. NLR was significantly related to anastomotic leakage and was an independent risk factor for anastomotic leakage. NLR was the only score for predicting the risk of anastomotic leakage that could be calculated preoperatively. There are many risk factors reported contributing to anastomotic leakage, such as anastomotic 
level from the anal verge, comorbidity, high ligation of the inferior mesenteric artery, male sex, and intraoperative complications [35].

The number of patients in the present study was small, therefore, further studies are in larger numbers of patients are needed. However, our results suggest that NLR can be used to predict the risk of anastomotic leakage preoperatively and may be helpful in determining the need for surgical procedures such as a diverting stoma.

\section{Conclusions}

Five types of risk evaluation scores were useful for predicting perioperative complications in patients with rectal cancer who received radical surgery. E-PASS CRS and NLR were risk scores related to anastomotic leakage. NLR was the only score for predicting the risk of anastomotic leakage that could be calculated preoperatively, suggesting that it is useful for assessing the need for a diverting stoma.

\section{Compliance with ethical standards}

Conflict of interest All authors have no conflict of interest to declare in association with this study.

Open Access This article is distributed under the terms of the Creative Commons Attribution 4.0 International License (http://creativecommons.org/licenses/by/4.0/), which permits unrestricted use, distribution, and reproduction in any medium, provided you give appropriate credit to the original author(s) and the source, provide a link to the Creative Commons license, and indicate if changes were made.

\section{References}

1. Siegel R, Naishadham D, Jemal A (2013) Cancer statistics. CA Cancer J Clin 63:11-30. Accessed 24 Oct 2016

2. Tevis ES, Kohlnhofer MB, Stringfield S et al (2013) Postoperative complications in patients with rectal cancer are associated with delays in chemotherapy that lead to worse disease free and overall survival. Dis Colon Rectum 56:1339-1348

3. Khuri FS, Henderson WG, DePalma GR et al (2005) Determinants of long term survival after major surgery and the adverse effect of postoperative complications. Ann Surg 242:326-341

4. Hendren S, Birkmeyer DJ, Yin H et al (2010) Surgical complication are associated with omission of chemotherapy for stage3 colorectal cancer. Dis Colon Rectum 53:1587-1593

5. Krarup PM, Nordholm CA, Jorgensen LN et al (2014) Anastomotic leak increases distant recurrence and long term mortality after curative resection for colonic cancer. Ann Surg 259:930-938

6. Aritinyan A, Orcutt TS, Anaya AD et al (2015) Infectious postoperative complications decrease long term survival in patients undergoing curative surgery for colorectal cancer. A study of 12,075 patients. Ann Surg 261:497-505
7. Law WL, Choi HK, Lee YM et al (2007) Anastomotic leakage is associated with poor long-term outcome in patients after curative colorectal resection for malignancy. J Gastrointest Surg 11:8-15

8. Henneman D, Ten Berge GM, Sniider SH et al (2014) Safety of elective colorectal cancer surgery: non surgical complications and colectomies are targets for quality improvement. J Surg Oncol 109:567-573

9. Pendlimari R, Cima RR, Wolff GB et al (2012) Diagnoses influence surgical site infections(SSI) in colorectal surgery: a must consideration for SSI reporting programs. J Am Coll Surg 2012 214:574-580

10. Branagan G, Finnis D (2005) Prognosis after anastomotic leakage in colorectal surgery. Dis Colon Rectum 48:1021-1026

11. NCCN guideline, Version 2. http://www.ncen.org/professionals/ physician_gls/f_guidelines.asp. Accessed 24 Oct 2016

12. Haga Y, Ikei S, Ogawa M (1999) Estimation of physiologic ability and surgical stress as a new prediction scoring system for postoperative morbidity and mortality following elective gastrointestinal surgery. Surg Today 29:219-225

13. Gawande AA, Kwaan MR, Regenbogen SE et al (2007) An Apgar score for surgery. J Am Coll Surg 204:201-208

14. Onodera T, Goseki N, Kosaki G (1984) Prognostic nutritional index in gastrointestinal surgery of malnourished cancer patients. Nihon Geka Gakkai Zasshi 85:1001-1005

15. Walsh SR, Cook EJ, Goulder F et al (2005) Neutrophil-lymphocyte ratio as a prognostic factor in colorectal cancer. J Surg Oncol 91:181-184

16. Copeland GP, Jones D, Walters M (1991) POSSUM a scoring system for surgical audit. Br J Surg 78:355-360

17. Tekkis PP, Prytherch DR, Kocher HM et al (2004) Development of a dedicated risk adjustment scoring system for colorectal surgery (colorectal POSSUM). Br J Surg 91:1174-1182

18. Dindo D, Demartines N, Clavien PA (2004) Classification of surgical complications. Ann Surg 240:205-213

19. Mc Dermott FD, Heeney A, Kelly ME et al (2015) Systematic review of preoperative, intraoperative and postoperative risk factors for colorectal anastomotic leaks. Br J Surg 102:462-479

20. Law WI, Chu KW, Ho JW et al (2000) Risk factors for anastomotic leakage after low anterior resection with total mesorectal excision. Am J Surg 179:92-96

21. Sadahiro S, Suzuki T, Ishikawa K et al (2001) Intraoperative radiation therapy for curatively resected rectal cancer. Dis Colon Rectum 44:1689-1695

22. Tominaga T, Takeshita H, Takagi K et al (2016) E-PASS scores as a useful predictor of postoperative complications and mortality after colorectal surgery in elderly patients. Int J Colorectal Dis 31:217-225

23. Ariake K, Ueno T, Takahashi M et al (2014) E-PASS comprehensive risk score is a good predictor of postsurgical mortality from comorbid disease in elderly gastric cancer patients. J Surg Oncol 109:586-592

24. Haga Y, Ikei S, Wada Y et al (2001) Evaluation of an estimation of physiologic ability and surgical stress scoring system to predict postoperative risk: a multicenter prospective study. Surg Today 31:569-574

25. Haga Y, Wada Y, Takeuchi $\mathrm{H}$ et al (2011) Prediction of anastomotic leak and its prognosis in digestive surgery. World J Surg 35:716-722

26. Mohri Y, Inoue Y, Tanaka K et al (2013) Prognostic nutritional index predicts postoperative outcome in colorectal cancer. World J Surg 37:2688-2692

27. Tokunaga R, Sakamoto Y, Nakagawa S et al (2015) Prognostic nutritional index predicts severe complications, recurrence, and poor prognosis in patients with colorectal cancer undergoing primary tumor resection. Dis Colon Rectum 58:1048-1057

28. Watt MB, Martin JC, Park JH et al (2015) Neutrophil count is the most important prognostic component of the differential 
white cell count in patients undergoing elective surgery for colorectal cancer. Am J Surg 210:24-30

29. Kubo T, Ono S, Ueno $\mathrm{H}$ et al (2014) Impact of the perioperative neutrophil-to-lymphocyte ratio on long term survival following an elective resection of colorectal carcinoma. Int J Colorectal Dis 29:1091-1099

30. Li MX, Liu XM, Zhang XF et al (2014) Prognostic role of neutrophil-to-lymphocyte ratio in colorectal cancer: a systematic review and meta-analysis. Int J Cancer 134:2403-2413

31. Cook EJ, Walsh SR, Farooq N et al (2007) Postoperative neutrophil-to-lymphocyte ratio predicts complications following colorectal surgery. Int J Surg 5:27-30

32. Forget P, Dinant V, Kock MD (2015) Is the neutrophil-to-lymphocyte ratio more correlated than $\mathrm{C}$-reactive protein with postoperative complications after major abdominal surgery? Peer J 3:e713. doi:10.7717/peerj.713

33. Regenbogen SE, Bordeianou L, Hutter MW et al (2010) The intraoperative surgical Apgar score predicts postdischarge complications after colon and rectal resection. Surgery 148:559-566

34. Miki Y, Tokunaga M, Tanizawa Y et al (2014) Perioperative risk assessment for gastrectomy by surgical Apgar score. Ann Surg Oncol 21:2601-2607

35. Trencheva K, Morrissey KP, Wells M et al (2013) Identifying important predictors for anastomotic leak after colon and rectal resection: prospective study on 616 patients. Ann Surg 257:108-113 